\title{
Implementación de un modelo de reconocimiento visual para mejorar el modelo adaptativo en niños con daltonismo usando un robot Nao para niños vulnerables en una ciudad inteligente
}

\author{
Tania Olivier ${ }^{1}$, Martha Jiménez-Del Real ${ }^{2}$, Mariam Escobedo ${ }^{2}$, \\ Ricardo Estrada-Medrano ${ }^{2}$, Alberto Ochoa ${ }^{1}$, Bruno Castro $^{3}$, Erwin Martínez ${ }^{1}$, \\ Salvador Noriega ${ }^{1}$ \\ ${ }^{1}$ Universidad Autónoma de Ciudad Juárez, Doctorado en Tecnología, \\ México \\ ${ }^{2}$ Universidad Autónoma de Ciudad Juárez, Ingeniería en Biomédica, \\ México \\ ${ }^{3}$ Universidad Autónoma de Ciudad Juárez, Maestría en Cómputo Aplicado, \\ Laboratorio Nacional de Tecnologías de Información, \\ México \\ tania.olivier@itlab.com, \{al148887, al148888\}@alumnos.uacj.mx, \\ \{alberto.ochoa, emartine\}@uacj.mx
}

\begin{abstract}
Resumen. Usando un robot humanoide Nao, proponemos apoyar a niños que tienen una deficiencia de color específico llamado "daltonismo" que evita que este grupo minoritario esté alerta de posibles advertencias visuales en juegos, parques y zoológicos. La relevancia de nuestro estudio radica en el apoyo en situaciones de peligro por parte de niños con daltonismo, identificar amenazas con colores específicos y ayudar a los niños con daltonismo en entornos visuales, como los entornos asociados con ciudades inteligentes.
\end{abstract}

Palabras clave: Niños con ceguera del color, reconocimiento de patrones, robot humanoide NAO.

\section{Implementation of a Visual Recognition Model to Improve the Adaptative Model in Children with Color Blindness Using a NAO Robot to Vulnerable Children in a Smart City}

\begin{abstract}
Using a Humanoid robot Nao, we propose support to children whom have a specific color deficiency named "Color Blindness", which prevents this minority group to be alert of possible visual warning in games, parks and zoos. The relevance of our study lies in the support in situations of danger on the part of children with color blindness, identifying threats with specific colors and to
\end{abstract}


Tania Olivier, Martha Jiménez-Del Real, Mariam Escobedo, Ricardo Estrada-Medrano, et al.

help children with color blindness in highly visual environments, such as the environments associated with smart cities.

Keywords: Color blindness children, pattern recognition, NAO humanoid robot.

\section{Introducción}

El sentido de la vista en los humanos como en otros organismos depende de sus ojos, usa dos tipos de células para la percepción de imágenes, bastones y conos [1].

Los polos permiten identificar la luminosidad, es decir, la cantidad de luz que se recibe del entorno y los conos permiten identificar el color o la frecuencia en el espectro de luz recibido [2].

En la mayoría de las personas hay tres tipos de conos, cada uno para percibir un color básico, estos pueden ser rojos, verdes o azules y los otros colores que se generan son el resultado de las diversas combinaciones que se reciben de la luz cantidades en sintonía con las frecuencias de estos colores básicos [3].

El mundo que nos rodea está diseñado para trabajar con colores que se perciben con tres conos, ya que la mayoría de las personas puede percibir el entorno con tres colores básicos, es decir, son tricromáticos, sin embargo, hay datos de personas con un cuarto tipo de cono, que les permite percibir más colores que la persona promedio visualiza, sin embargo, estas personas generalmente tienen problemas para describir el ambiente y los tonos que perciben, ya que el mundo no está hecho contemplando sus percepciones sensoriales [3].

Por otro lado, también hay casos de personas con una percepción de color por debajo del promedio, esta condición se llama daltonismo y se considera una discapacidad promedio, ya que los colores con percepción tricromática son utilizados en diversas actividades, como identificar objetos en una conversación, etiquetar situaciones peligrosas, saber cuándo avanzar en un semáforo, decidir qué ropa comprar y disfrutar de las formas de arte como la pintura o la fotografía [4].

El daltonismo se puede clasificar en cuatro variantes de acuerdo con los conos que se cuentan para percibir el medio ambiente, estas variantes pueden ser tricromáticas, dicromáticas y monocromáticas anómalas o acromatopsia [1].

La variante más común de daltonismo es la tricromacia anómala, en la que todos los conos son disponible para la percepción del color, pero hay una deficiencia en algunos de los conos, a su vez según su gravedad se puede separar en tricromacia leve anómala, media o fuerte y dependiendo del color en el que la deficiencia ocurre [4], la tricromacia se puede dividir en deuteranomalía o deficiencia en la percepción de verdes, protanomaly en la percepción de rojos y tritanomacia en la percepción del cono azul [3].

Otra variante del daltonismo que ocurre con menos frecuencia, pero de mayor gravedad es la dicromía, en este, un tipo de cono está ausente, es decir, la persona no puede percibir uno de los colores básicos, lo que causa el persona que tiene problemas con todos los colores que tienen este tono en su constitución, por ejemplo, una persona que tiene problemas con el cono verde, tendrá problemas con todos los tonos de verdes, pero también el amarillo y el café que en cierta medida está constituido con el color verde como base. La dicromacia también puede ser clasificada según el cono ausente, 
Implementación de un modelo de reconocimiento visual para mejorar el modelo adaptativo...

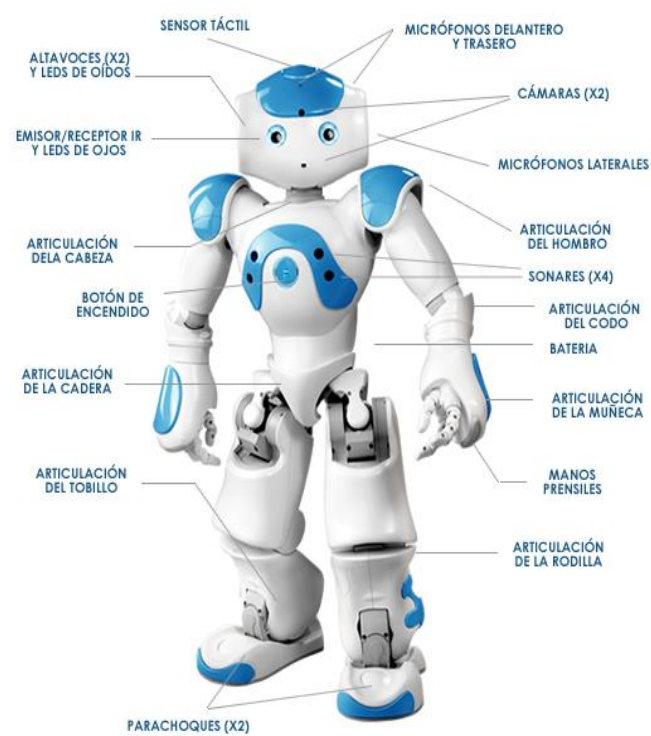

Fig. 1. Componentes asociados con un humanoide Robot NAO [5].

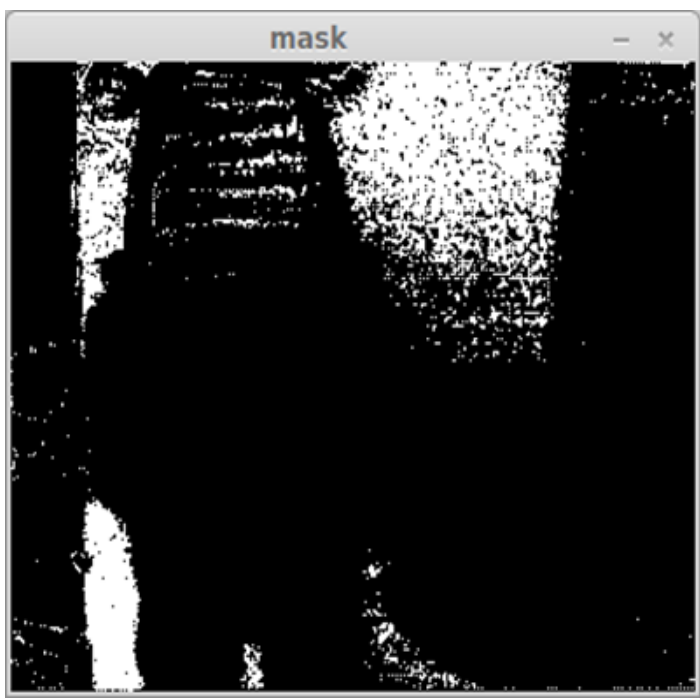

Fig. 2. Mascara que resalta en color blanco los colores verdes.

es deuteranopia cuando el cono verde está ausente, la protanopia con el cono rojo ausente y tritanopia cuando el cono azul está ausente [3].

Uno de los aspectos más importantes de una Ciudades inteligentes es la inclusión de sus ciudadanos, razón por la cual esta investigación tiene gran relevancia porque busca ayudar a los niños con daltonismo a comprender la relevancia de los colores en todos los días de la vida [2]. 
Materiales y métodos de la investigación. Usamos un humanoide Robot NAO para lograr nuestro objetivo de determinar los colores y poder ayudar a los niños que tener problemas de daltonismo.

Componentes de la NAO. Cada aspecto del humanoide Robot NAO tiene diferentes dispositivos asociados para poder identificar a través de una cámara especializada para poder visualizar imágenes y, por lo tanto, realizar un procesamiento del color, los diferentes los dispositivos asociados con el humanoide Robot NAO se describen en la figura 1 .

\section{Metodología aplicada}

Seguimos un proceso adecuado para ayudar a los niños con daltonismo, primero la importancia del proceso era capturar la imagen que se estaba viendo en el momento actual (Fig. 2), luego convertimos la imagen a un proceso capaz de ser analizado, en nuestro caso programamos el código asociado con la correcta identificación del color verde, luego eliminamos los problemas de ruido y finalmente analizamos si el objeto que deseamos alcanzar ayuda a un niño con daltonismo es adecuado para nuestro propósito de investigación. Detallaremos cada uno de los procesos llevados a cabo y finalmente mostraremos el código asociado con todo el proceso.

Capturar imagen. Cargamos las librerías cv2 y numpy. Activa la cámara y guarda lo que puedes registrar a través de tu dispositivo.

import cv2

import numpy as $n p$

captura $=c v 2$. VideoCapture $(0)$

Convierte la imagen. Creamos un ciclo infinito (while (1) o while (true)). En el interior, leemos un cuadro y lo guardamos dentro de una variable que llamaremos 'imagen', luego convertiremos este cuadro a HSV, ya que es más fácil analizar imágenes en este color modelo.

while(1):

_, imagen = captura.read ()

$h s v=c v 2 . c v t$ Color(imagen, $\left.c v 2 . C O L O R \_B G R 2 H S V\right)$

Identificación de objetos verdes. Necesitamos dos matrices para almacenar el rango de colores que detectamos. El límite inferior será 49, 50, 50, un oscuro verde. El límite superior será $80,255,255$, un verde marino muy claro. Nuestro programa detectará todos los colores dentro de este rango.

verde_bajos $=$ np.array $([49,50,50])$

verde_altos $=$ np.array $([80,255,255])$

Uno de los principales problemas para un niño daltónico es precisamente la identificación de objetos verdes. Necesitamos saber qué píxeles de la imagen están dentro del rango. Para esto crearemos una máscara que almacena ellos. 
Implementación de un modelo de reconocimiento visual para mejorar el modelo adaptativo...

Pero, ¿qué se propone usar una máscara? Es una imagen que contiene solo dos colores: blanco y negro. En nuestro caso, pintará los píxeles verdes en blanco y el resto en negro. Por ejemplo, la imagen que puse en el principio es una máscara que detecta verde. mask $=$ cv2.inRange (hsv, verde_bajos, verde_altos)

Eliminar el ruido. Necesitamos descartar todos aquellos objetos que no alcanzan cierto tamaño (ruido).

Para comenzar, calcularemos el momento de los objetos que hemos detectado. La función cv2.moments () nos da como resultado un diccionario. Estamos interesados en la clave 'm00', que ahorra el valor del área del momento:

moments $=c v 2$. moments $($ mask $)$

area $=$ moments ['m00']

Para eliminar el ruido, permaneceremos solo con aquellos objetos cuya área excede un cierto valor. por esto usaremos un condicional. Después de jugar con diferentes números, se determinó que 2000000 es el valor para esta investigación.

Buscamos el centro del objeto en cuestión y mostramos sus coordenadas en la pantalla. Para visualizarlo en la imagen dibujaremos un pequeño rectángulo rojo:

If (area > 2000000):

\#Buscamos los centros

$x=\operatorname{int}($ moments ['m10']/moments ['m00'])

$y=\operatorname{int}$ (moments['m01']/moments['m00'])

\#Escribimos el valor de los centros

print " $x=", x$

print " $y=", y$

\#Dibujamos el centro con un rectángulo

cv2.rectangle(imagen, $(x, y),(x+2, y+2),(0,0,255), 2)$

Mostrar imagen. Mostraremos dos ventanas. En el primero, la imagen original aparecerá con el centro del objeto. En el segundo será la máscara en blanco y negro.

cv2.imshow('mask', mask)

cv2.imshow('Camara', imagen)

tecla $=$ cv2.waitKey $(5) \& O x F F$

if tecla $=27$ :

break

cv2.destroyAllWindows()

Aquí está el código completo que se ha escrito. Un aspecto relevante es que hemos adaptado el código inicial para que puede identificar objetos que tienen variantes de un cierto color y determinar si dos objetos de diferente los colores están asociados en un diorama.

\#Algoritmo de detección de colores

\#Por Glar3 
Tania Olivier, Martha Jiménez-Del Real, Mariam Escobedo, Ricardo Estrada-Medrano, et al.

\#Detecta objetos verdes, elimina el ruido y busca su centro

import cv2

import numpy as $n p$

\#Iniciamos la camara

captura $=c v 2$. VideoCapture $(0)$

while(1):

\#Capturamos una imagen y la convertimos de $R G B->H S V_{\_}$, imagen = captura.read()

$h s v=c v 2 . c v t$ Color(imagen, $\left.c v 2 . C O L O R \_B G R 2 H S V\right)$

\#Establecemos el rango de colores que vamos a detectar

\#En este caso de verde oscuro a verde-azulado claro

verde_bajos $=$ np.array $([49,50,50]$, dtype $=n p$. uint 8$)$

verde_altos $=n$ p.array $([80,255,255]$, dtype $=n p$. uint8 $)$

\#Crear una máscara con solo los pixeles dentro del rango de verdes

mask $=c v 2$. inRange $\left(h s v, v e r d e \_b a j o s, v e r d e \_a l t o s\right)$

\#Encontrar el area de los objetos que detecta la cámara

moments $=c v 2$. moments $($ mask $)$

area $=$ moments ['m00']

\#Descomentar para ver el área por pantalla

\#print area

if(area > 2000000):

\#Buscamos el centro $x$, y del objeto

$x=\operatorname{int}($ moments ['m10']/moments['m00'])

$y=\operatorname{int}($ moments ['m01']/moments['m00'])

\#Mostramos sus coordenadas por pantalla

print " $x=", x$

print " $y=", y$

\#Dibujamos una marca en el centro del objeto

cv2.rectangle(imagen, $(x, y),(x+2, y+2),(0,0,255), 2)$

\#Mostramos la imagen original con la marca del centro y la mascara

cv2.imshow('mask', mask)

cv2.imshow('Camara', imagen)

tecla $=$ cv2.waitKey $(5) \& O x F F$

if tecla $==27$ :

break

cv2.destroyAllWindows() 


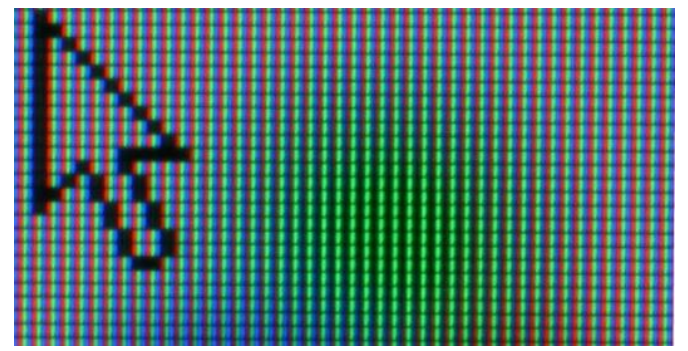

Fig. 3. Imagen de monitor de computadora.

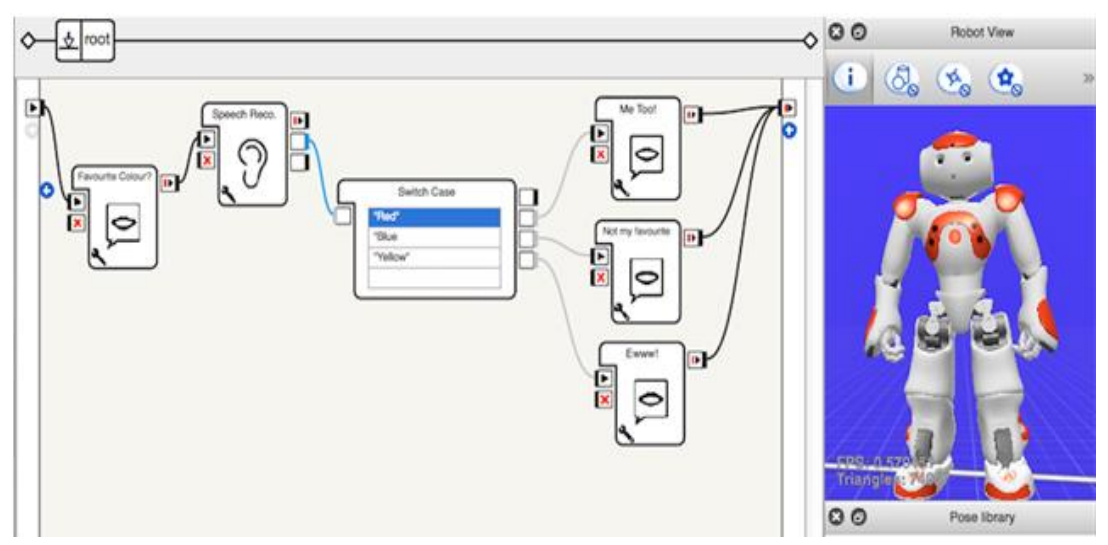

Fig. 4. Representación de modelo de reconocimiento de color codificado en el software Coreographe.

Un aspecto relevante de esta investigación es que el código vinculado al dispositivo puede leer e interpretar una gama más amplia de colores y que no solo es rojo, verde, azul y amarillo. Una situación relevante de esta investigación es usar el espacio de color HSV y las matrices de valores máximos y mínimos que utilizamos en OpenCV para detectar colores significados, decidimos agregar un código en Python que se obtuvo en la literatura para poder ajustar estos valores HSV en tiempo real con barras de desplazamiento, como lo propuesto en [3].

¿Cuál es el espacio de color HSV?

Cuando vemos un color en la pantalla, lo que estamos viendo en realidad son miles de píxeles que brillan con una cierta intensidad Cada píxel está formado por tres luces: una roja, una verde y una azul. La sensación de color se produce variando la intensidad de estas tres luces y alterando así la cantidad de rojo, azul y verde luz recibida por los ojos del usuario [3].

Dentro de la computadora, los colores están codificados por números. Hay varias formas de codificar colores. El más popular es el sistema de color RGB, que indudablemente todos ustedes conocen. Este sistema asigna a cada color una cantidad de Rojo, Verde y azul entre 0 y 255. Por ejemplo, rojo puro es [255,0,0].

Pero hay otras formas de codificar los colores. El HSV (Hue, Saturation, Value- Hue, Saturation y Value) es ideal para el reconocimiento de color. 


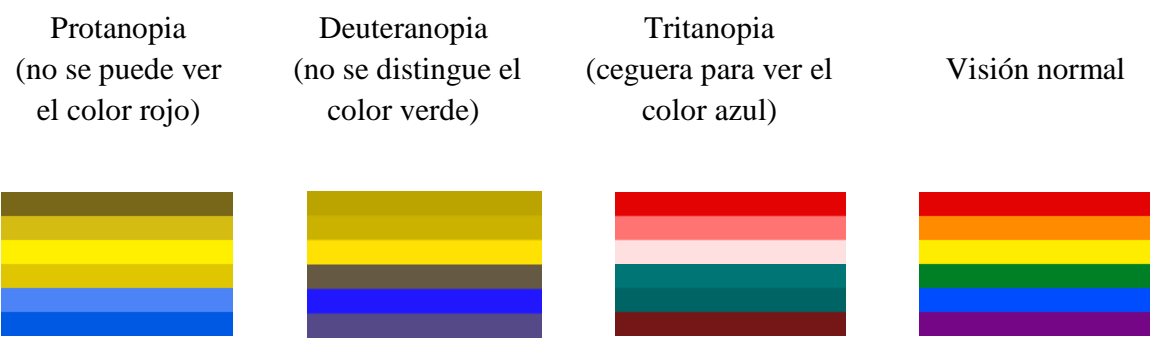

Fig. 5. Objetivo asociado con la adecuada reconstitución del color por nuestro Nao Robot [3].

Tabla 1. Porcentajes de incidencia para las diferentes variaciones de daltonismo en todo el mundo.

\begin{tabular}{cccc}
\hline Tipo & Denominación & Incidencia & H / M Ratio \\
\hline Monocromía & Acromatopsia & $0.00003 \%$ & \\
\hline Dicromacia & Deuteranopia & $1.27 \%$ & $0.01 \%$ \\
\hline Protanopia & $1.01 \%$ & $0.02 \%$ & \\
\hline Tritanopia & & $0.0001 \%$ & $0.36 \%$ \\
\hline $\begin{array}{c}\text { Tricromacia } \\
\text { Anómala }\end{array}$ & Deuteranomalía & $4.63 \%$ & $0.03 \%$ \\
\hline Protanomalia & & $1.08 \%$ & $0.0002 \%$ \\
\hline Tritanomacia & & \\
\hline
\end{tabular}

\section{Análisis de resultados}

Intentamos determinar los aspectos relevantes que ayudarían a los niños con daltonismo a ser capaces de identificar ciertos colores para él, usamos Coreographe para poder determinar las funciones apropiadas para realizar la identificación de un objeto de un color específico, como se puede ver en la figura 4.

Un aspecto relevante de nuestra investigación fue analizar adecuadamente las variaciones de colores y cómo el umbral de un objeto, para poder especificar si la relevancia de la tonalidad afectó el tiempo de respuesta para detectar un objeto específico, lo que podría ayudar en un aspecto concreto al niño con daltonismo [7] como en figura 5.

Monocromática o acromatopsia, es la condición más rara de daltonismo, en este, todos los conos están ausentes, por lo tanto, solo el ambiente se percibe en escalas de grises o brillo y, aunque es muy raro, representa una gran dificultad para las personas que la padecen, ya que no pueden llevar una vida normal sin la asistencia de personas sanas. Estas personas no pueden beber un líquido de una botella sin antes buscar una etiqueta que confirma su contenido, no pueden identificar si un alimento está en buenas condiciones antes de comerlo, pueden no elegir su ropa, identificar un espacio de estacionamiento, entre otras dificultades [5]. 

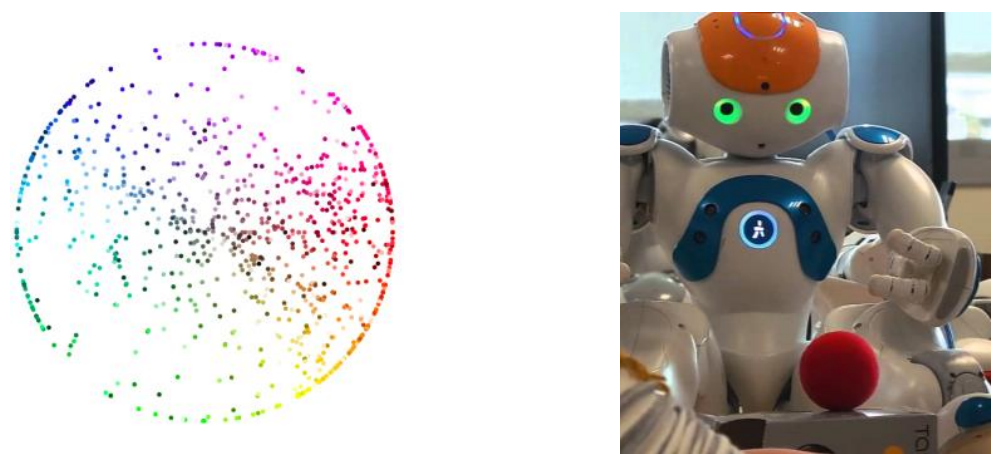

a)

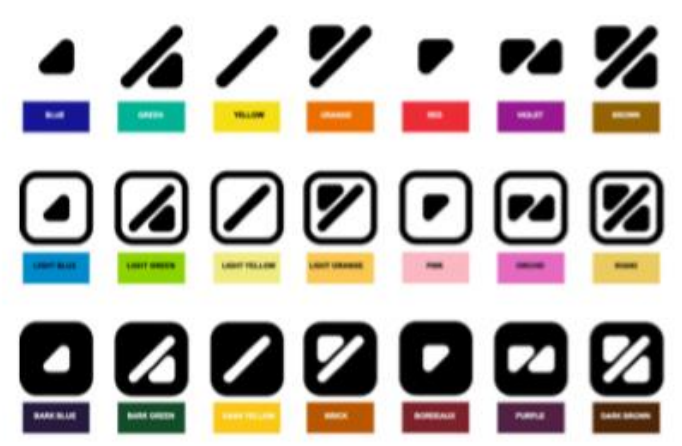

b)

Fig. 6. (a) Detección de color utilizando nuestro Robot NAO para apoyar a niños con ceguera. (b) Variaciones más comúnmente.

Aproximadamente el $10 \%$ de las personas sufre de alguna deficiencia de color o ceguera, es decir, alrededor de 700 millones de personas sufren del daltonismo, teniendo en cuenta que la población mundial supera los 7.000 millones de habitantes.

La Tabla 1 muestra los porcentajes de incidencia en hombres y mujeres para cada una de las variantes de daltonismo en todo el mundo.

Considerando la incidencia y llevando a cabo un diseño adecuado de experimentos, demostramos la mejora realizada para niños con daltonismo como se puede ver en la figura 6.

\section{Conclusiones}

Un robot humanoide NAO, puede ayudar a los niños con daltonismo a identificar correctamente los colores primarios y sus derivaciones porque sin la posibilidad de poder ver ese tipo de tonalidades, estarán expuestas a la información visual del peligro es por qué debería ser trabajar con un modelo de sonidos específicos para determinar 


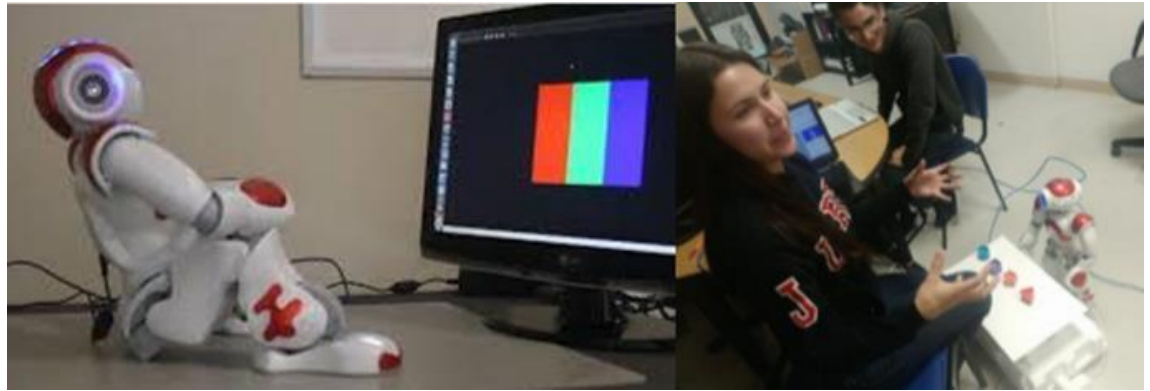

a)

b)

Fig. 7. a) Identificación en línea de diversos colores y sus variaciones para explicar la relevancia del peligro para el color niños con ceguera b) Diseño de experimentos en el Laboratorio LANTI.
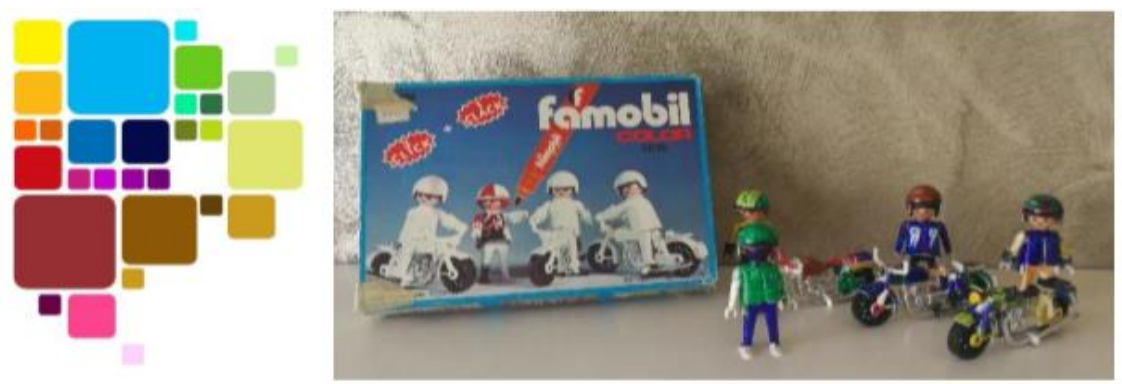

Fig. 8. Modelado de combinaciones innovadoras de color para apoyar el daltonismo para decorar clics sin color.

peligro en parques, zoológicos y lugares de atracciones nocturnas como parques infantiles [7].

Intentamos modelar la acomodación de objetos de diferentes tamaños y colores para determinar el sentido de organización, para apoyar a los niños con daltonismo para organizar actividades lúdicas con otros niños. Es muy importante para los niños conocer los colores reales y su relevancia en nuestras vidas, por este motivo definimos actividades relacionadas con el color y determinar el tiempo de gasto para cada tarea de agrupación [6].

\section{Investigación futura}

Un aspecto decisivo del trabajo futuro es condicionar nuestro modelo, para que pueda identificar inmediatamente un cambio de color en un escenario de peligro, como se puede ver en la figura 7. Se ha demostrado que un niño con esta condición tiempo para identificar la iconografía característica de cada color. La relevancia de este estudio es apoyar con dimensionalidad del color la interacción de los niños con ceguera al color con cualquier objeto, incluidos los juguetes que serán peligrosos si no analiza las 
Implementación de un modelo de reconocimiento visual para mejorar el modelo adaptativo...

especificaciones en color. Proponemos modelar el color en los juguetes de Playmobil utilizando nuestro software como se muestra en la figura 8 .

\section{Referencias}

1. Boukezzoula, R., Coquin, D., Nguyen, T.L., Perrin, S.: Multi-sensor information fusion: Combination of fuzzy systems and evidence theory approaches in color recognition for the NAO humanoid robot. Robotics and Autonomous Systems 100, pp. 302-316 (2018)

2. Nguyen, T. L.: Fusion d'informations multi-capteurs pour la commande du robot humanoïde NAO. (Multi-sensor information fusion: application for the humanoid NAO robot) (2017)

3. Kumar, A., Patel, A., Dwivedy, S. K.: Development of a NAO Humanoid based Medical Assistant. In: AIR'17 Proceedings of the Advances in Robotics 35, pp. 1-35, New Delhi, India (2017)

4. Bussey, D., Glandon, A., Vidyaratne, L., Mahbubul, A., Khan, M.: Iftekharuddin: Convolutional neural network transfer learning for robust face recognition in NAO humanoid robot. In: 2017 IEEE Symposium Series on Computational Intelligence (IEEE SSCI 2017), pp. 1-7 (2017)

5. Hu, Y., Sirlantzis, K., Howells, G., Ragot, N., Rodriguez, P.: An online background subtraction algorithm deployed on a NAO humanoid robot based monitoring system. Robotics and Autonomous Systems 85 (2016)

6. Fitter, N. T., Kuchenbecker, K. J.: Qualitative User Reactions to a Hand-Clapping Humanoid Robot. In: International Conference on Social Robotics, pp. 37-47 Springer, Cham (2016)

7. Singh, A. K., Nandi, G. C.: NAO humanoid robot: Analysis of calibration techniques for robot sketch drawing. Robotics and Autonomous Systems 79, pp. 108-121 (2016) 